\title{
Matrix Composition and Fine-scale Structure Analysis of NMC Li-ion Battery Using Atom Probe Tomography
}

Yimeng Chen ${ }^{1}$, Peter Clifton ${ }^{2}$ and Ty Prosa ${ }^{3}$

${ }^{1}$ CAMECA, Madison, Wisconsin, United States, ${ }^{2}$ Cameca, United States, ${ }^{3}$ CAMECA® Instruments Inc., 5470 Nobel Drive, Madison, WI 53711 USA, Wisconsin, United States

Li ion batteries are widely used for portable PCs, cellular phones and other portable electronics. A recent market forecast predicts a massive expansion in production driven by electric vehicles. An anode, a cathode and an electrolyte are the three main components in a Li-ion battery. Most cathode materials are made of a Li-based oxide to provide mobile Li ions. Lithium-cobalt-oxide comprised over $34 \%$ of the market share in 2018; however, lithium-nickel-manganese-cobalt (NMC) [1]. Its market share is expected to increase to $48 \%$ in 2025 from the current $19 \%$. The shift is already taking place by major manufactures owing to its in addition to cost competitiveness. NMC shows high capacity and power flow of 150-220 $\mathrm{Wh} / \mathrm{kg}$, a nominal voltage of 3.6V and a 1000-2000 cycle life [1]. Manufacturing R\&D also shows a trend of shifting to high $\mathrm{Ni}$ content systems for enhanced capacity. In this work we investigate the composition and fine-scale microstructure analysis of two types of NMC cathode materials. Cathode tapes with $90 \%$ active particle, $5 \%$ binder and 5\% conductive carbon were obtained from NEI Corporation (product names EB-50E-622 and EB-50E-811). The nominal compositions are Li(NixMnyCoz)O2 X:Y:Z=6:2:2 and $\mathrm{X}: Y: Z=8: 1: 1$. SEM and EDS analysis on these materials confirms the particles are $\sim 10$ um in diameter and compositionally homogeneous at the sub-micron scale. Standard atom probe tomography specimen preparation method with focused ion beam lift-out was used for analysis of the sub surface region on various particles. On average each lift-out region extracts five atom probe specimens. Multiple particles from each sample were examined to better understand particle to particle variation. Analysis conditions for data collection were systematically studied and optimized to balance data quality and yield. A typical mass spectrum exhibits the constituent elements where $\mathrm{Ni}$, Co and Mo are favorable to form molecular ions with oxygen (Figure 1a). Corresponding peaks for $\mathrm{Ni}$ and $\mathrm{Co}$ may interfere with each other due the presence of hydride species. Peak decomposition was used to obtain the best accuracy composition by deconvolution the overlapping species. The overall matrix/bulk compositions of three particles from NMC622 and two particles from NMC811 are reported in Figure 1b. The compositional variation is well below the +/-2 sigma statistic error calculated from each population indicating relatively good homogeneity from specimen to specimen and particle to particle. Based on the results, the compositions of NMC622 and NMC811 are easily differentiated using atom probe. Atom probe is also ideal for studying fine-scale features. A variety of features in a range of morphologies and size were observed including primary particle boundaries and secondary phases. Figure 2 shows an example feature with significant $\mathrm{Li}$ enrichment up to 40 at.\% (compared to matrix values of 27 at.\%). The same type of composition fluctuation has been identified as the Li2MO3 phase in the cathode after charging cycles using atom probe $[2,3]$. In conclusion, atom probe has been shown to provide excellent results with NMC cathode material for batteries. It is capable identifying of the matrix composition and of study of fine-scale compositional variations. The two types of NMC may be quantitively and precisely differentiated using their Li to metal and metal to metal ratios (Figure $1 b$ ). 

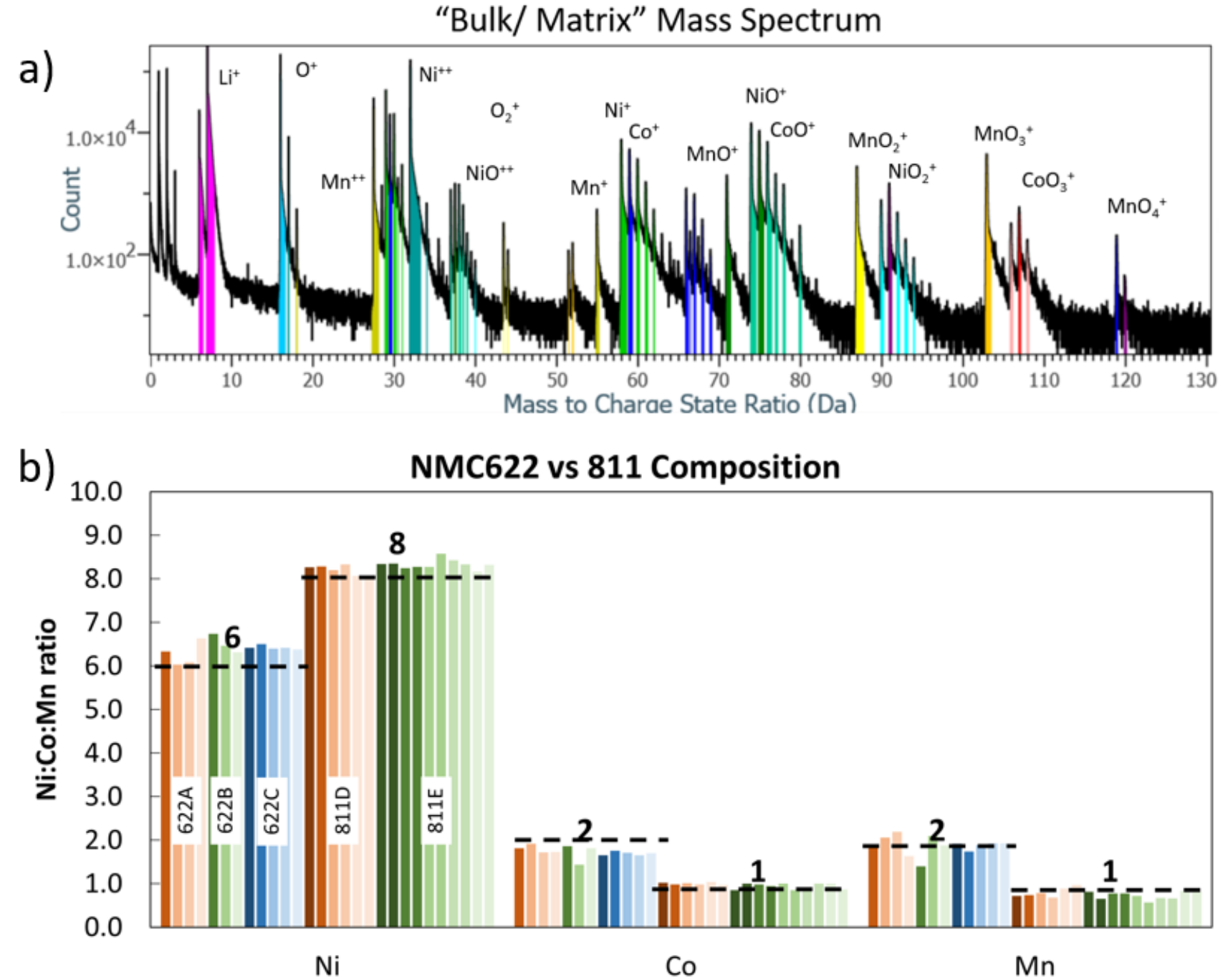

Figure 1. Figure 1a) Example of NMC mass spectrum b) Composition of NMC 622 particle A, B and C along with NMC811 particle D and E. The dashed lines indicate materials' nominal composition.
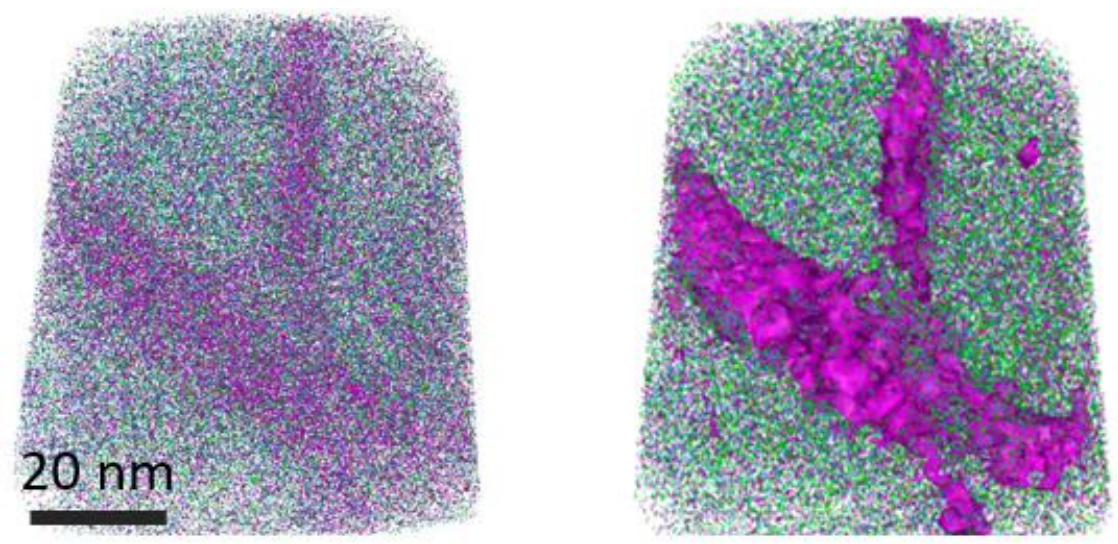

Figure 2. Figure 2. Atom map showing Li rich feature in purple in NMC622. Right is Li rich feature highlighted with 35 at.\% Li isosurface. 


\section{References}

[1] Worldwide Rechargeable Battery Market 2019-2030, Avicenne Energy, $28^{\text {th }}$ edition.

[2] A. Devaraj, et al. Nature Communications 6.1 (2015): 1.

[3] J.Y Lee, et al. Journal of Power Sources 379 (2018): 160. 